\title{
Within and between session changes in subjective and neuroendocrine stress parameters during magnetic resonance imaging: A controlled scanner training study
}

\author{
Ulrike Lueken $^{\mathrm{a}, \mathrm{b}, 1,{ }^{*} \text {, Markus Muehlhan }{ }^{\mathrm{a}, \mathrm{b}, \mathrm{c}, 1} \text {, Ricarda Evens }}{ }^{\mathrm{a}, \mathrm{b}}$, Hans-Ulrich Wittchen ${ }^{\mathrm{a}, \mathrm{b}}$, \\ Clemens Kirschbaum ${ }^{\mathrm{c}}$ \\ a Institute of Clinical Psychology and Psychotherapy, Department of Psychology, Technische Universität \\ Dresden, Dresden, Germany \\ b Neuroimaging Center, Department of Psychology, Technische Universität Dresden, Dresden, Germany \\ c Chair of Biopsychology, Department of Psychology, Technische Universität Dresden, Dresden, Germany
}

\section{Summary}

Accumulating evidence suggests that the magnetic resonance imaging (MRI) scanner can act as a stressor, eliciting subjective and neuroendocrine stress responses. Approaches to familiarize subjects with the scanner could help minimizing unintended effects on neural activation patterns of interest. Controlled studies on the effects of a scanner training are however missing. Using a comparative design, we analyzed within- and between session changes in subjective and neuroendocrine stress parameters in 63 healthy, scanner-naïve adults who participated in a two-day training protocol in an MRI, mock, or lab environment. A habituation task was used to assess within-session changes in subjective and neuroendocrine (cortisol) stress parameters; between-session changes were indicated by differences between days. MRI and mock, but not lab training were successful in reducing subjective distress towards the scanner. In contrast, cortisol reactivity towards the training environment generally increased during day 2, and the percentage of cortisol responders particularly rose in the mock and MRI groups. Within-session habituation of subjective arousal and anxiety was observed during both days and irrespective of training condition. Present findings demonstrate that training in a scanner environment successfully reduces subjective distress, but may also induce sensitization of endocrine stress levels during repeated scanning. Subjective distress can further be stabilized by acclimating subjects to the environment prior to the MRI assessment, including a short habituation phase into the assessment protocol. If replicated, present findings should be considered by researchers employing repeated measurement designs where subjects are exposed to a scanner more than once.

Keywords: fMRI; Scanner; Mock; Anxiety; Cortisol; Stress; Training; Habituation; Familiarization

\section{Introduction}

Over the past 20 years the use of functional magnetic resonance imaging (fMRI) as a tool in neuroimaging research has increased continuously. But despite the advantages this noninvasive technique offers, the scanner setting itself can elicit subjective distress, severe claustrophobia or even panic attacks in a substantial portion of patients (Fishbain et al., 1988; Dewey et al., 2007; Spiegelhalder et al., 2009). Recent evidence also suggests elevated 
endocrine stress parameters in response to the scanner, including increased activity of the hypothalamic-pituitary-adrenal (HPA) and sympatho-adrenal-medullary (SAM) axis (Tessner et al., 2006; Eatough et al., 2009; Muehlhan et al., 2011). It is thus plausible to assume that scanner-related distress might act upon neural activation patterns of interest in fMRI studies (Muehlhan et al., 2011).

Exploring the changes in subjective and neuroendocrine stress reactions, consequences emerging within a single scanner session may differ from those seen in repeated sessions. As outlined by Chapman et al. (2010), within-session changes due to habituation processes could interact with the order of tasks or confound resting state investigations. Between-session changes on the other hand could affect repeated measurement designs, which are often used in clinical studies. Following this, the detection of treatment-related neurofunctional changes could at least be partly confounded by changes in state anxiety towards the scanner environment, particularly in vulnerable patient groups such as anxiety patients. Moreover, comparisons between (distressed) patients and healthy controls could be biased, since both groups may differ in their stress levels (Lueken et al., 2011). Assuming such relationships between the stress-eliciting properties of the scanner and neural activation patterns of interest, the development and evaluation of protocols to familiarize subjects with the scanner and minimize individual stress responses could offer an incremental value in controlling these unintended effects and thus in improving data quality.

Evidence suggests that psychological interventions such as information and counselling, cognitive distraction strategies or relaxation are effective in reducing subjective anxiety during scanning (Quirk et al., 1989; Grey et al., 2000). Simulation or mock scanners represent another promising approach used quite frequently to familiarize children to the fear inducing features of the scanner environment. The mock scanner can act as a sufficient MRI scanner surrogate in eliciting subjective and physiological signs of claustrophobic fear (McGlynn et al., 2003, 2007). Furthermore, elevations in cortisol during mock training have been reported for vulnerable patient groups (e.g. children with autism spectrum disorder; Corbett et al., 2006), but not for adolescents in general (Eatough et al., 2009). However, potential carry-over effects on cortisol levels between successive mock and MRI scanning cannot be excluded in the study from Eatough et al. (2009). Findings regarding between-session changes subsequent to a mock or MRI session are inconclusive so far, with many studies lacking sufficient control conditions or multimodal assessment of subjective and physiological stress parameters. Satisfactory MRI pass rates and data quality could be obtained in children after a mock training session, reducing the need for sedation (De Bie et al., 2010). In line with this, subjective anxiety, cortisol values and heart rates have been reported to decrease in adults during repeated MRI scanning (Chapman et al., 2010; Peters et al., 2011). However, mock scanning does not necessarily prevent endocrine responses to the actual MRI scan (Eatough et al., 2009), and return of fear after successful behavioural exposure to a mock has been reported in about one third of claustrophobic patients (Wood and McGlynn, 2000).

Employing a comparative design using a lab, mock, or MRI training environment, the aim of this study was to analyze within- and between session changes in subjective and neuroendocrine stress parameters during a two-day scanner training protocol in healthy, scanner-naïve adults. We hypothesized that both mock and MRI training would induce distress: subjects in the mock and MRI training group would be more stressed on day 1 than the lab group. Further, we hypothesized that both mock and MRI training would be successful in reducing distress towards the MRI setting: compared to day 1, decreased stress levels should be observed during day 2 in the former two groups, while stress levels should increase in the lab group. Within-session habituation should occur in all subjects during day 1 in 
response to the scanner setting (mock and MRI group), or as a general reaction to the novel research environment (lab group). During day 2, however, within-session habituation should be observable in the lab group only as a response to the novel scanner setting.

\section{Methods}

\subsection{Subjects and procedure}

The sample included 70 right-handed, healthy, scanner naïve students, aged between 18 and 35 years. Subjects were recruited via posters and flyers at the Technische Universität Dresden. Exclusion criteria included smoking, oral contraceptives, body mass index below 18 or above 26 , any history of acute or chronic medical disease or failure to meet basic MRI compatibility. One subject had to be excluded due to previous scanner experience; six subjects could not attend the second testing day, reducing the final sample to $n=63$ subjects ( 34 males). Subjects received course credits or an expense allowance of s20. The study protocol was approved by the local ethics committee.

The study was conducted at the Neuroimaging Center of the Technische Universität Dresden and comprised two testing days with each session lasting $1.5 \mathrm{~h}$. The training protocol was comparable on both days, but the setting was manipulated on day 1 . At day 1 , subjects were randomly allocated to one of the three experimental conditions (lab training, mock training, MRI training), while on day 2 the training was repeated in the MRI scanner for all subjects. The mock scanner was built upon an old, not in use CT scanner that was enlarged with a tunnel resembling the MRI tomograph. It was equipped with a manually operated patient table, replica of a head coil, head phones, and a visual back projection system so that subjects could conduct the tasks as if they were in the MRI scanner. Sounds of structural localizer and echo-planar imaging (EPI) sequences were transmitted via headphones in order to mimic the actual MRI session as closely as possible. For the MRI condition a 3-T Trio-Tim MRI wholebody scanner (Siemens, Erlangen, Germany) was used. To minimize effects due to different MRI staff, personnel was kept constant. Fig. 1 illustrates the different settings.

Assessments were scheduled between $1100 \mathrm{~h}$ and $1500 \mathrm{~h}$ to avoid a bias in cortisol levels due to circadian rhythm (Weitzman et al., 1971). Subjects were instructed to refrain from eating and drinking $1 \mathrm{~h}$ before testing. After obtaining informed consent, but prior to allocation to the experimental conditions, state affect ratings were collected using the Multidimensional Mood Questionnaire (MDMQ, German version: Steyer et al., 1997). Subjects then received training on a working memory task (n-back; data not reported here) at both testing days in order to obtain stable performance levels. Afterwards, subjects were allocated to their respective setting: the MRI training took place in the MRI scanner, the mock training in the mock scanner and subjects of the lab condition stayed in the preparation room. The training protocol included a habituation task (12 $\mathrm{min})$ in order to assess within-session mood changes (subjective valence, arousal, and anxiety at the beginning, after four, eight and $12 \mathrm{~min}$ ) in the respective condition. During habituation subjects were instructed to lie calmly in the MRI, mock or sit in front of the lab computer and to complete the ratings. In the MRI and mock condition, subjects were scanned or a scan was simulated, respectively. Each session consisted of two runs and started with the habituation task followed by the n-back task. At the end of each session subjects completed several questionnaires. Six saliva samples were taken during each day (T1: after the informed consent (min 10), T2: after working memory training session ( $\min 35$ ), T3: immediately before the habituation task (min 55), T4: after the habituation task (min 70), T5: after the working memory task (min 87), T6: after completion 
of the questionnaires (min 110)). Within-session changes were assessed during the habituation task at the beginning of the scanner session. Between-session changes were investigated examining differences between day 1 and day 2 .

\subsection{Measures}

Scanner tasks. To assess within-session changes in subjective mood a habituation task was implemented at the beginning of the training session. After participants entered their specific setting they were asked to watch a white cross in the middle of the monitor for $12 \mathrm{~min}$. Subjects rated their emotional state via button-press every four min for a total of four times (T1-T4). The three dimensions valence, arousal and anxiety were measured on a 9-point Likert scale (valence: „I feel bad (1) to good (9)“, arousal: „I am nervous not at all (1) to extremely (9)“; anxiety: „I am anxious not at all (1) to extremely (9)“). In order to assess working memory capacity an n-back task was used to manipulate the working memory load with a 2-back and a 3-back condition (total duration: 14:50 min; behavioural and fMRI data will be reported elsewhere). Both tasks were programmed with Presentation ${ }^{\circledR} 11.3$ (Neurobehavioral Systems, Inc., CA) and presented digitally during the training session.

State and trait affectivity. To assess differences in mood at the beginning of each session, subjects completed the short form of the MDMQ after the informed consent. It consists of 12 items assessing three mood dimensions (valence: good mood, bad mood; alertness: awake, tired; calmness: calm, nervous). Several questionnaires were used to characterize trait affectivity including depressive symptoms (Beck Depression Inventory: BDI II, Hautzinger et al., 2006), general anxiety (Stait Trait Anxiety Inventory: STAI, Laux et al., 1981; Anxiety Sensitivity Index: ASI, Hoyer and Markgraf, 2003), and claustrophobic anxiety (Claustrophobia Questionnaire: CLQ, Radomsky et al., 2001). BDI II and CLQ scores were available for $\mathrm{n}=62$ subjects.

Biochemical determination of salivary cortisol and parametrization. Saliva samples were collected using Salivetten ${ }^{\circledR}$ (Sarstedt, Nümbrecht, Germany). Subjects were instructed to gently chew the swabs in order to stimulate saliva flow. Outside the scanner, swabs were directly passed to the subject by the study personnel. During the scanner session, subjects lying on the MRI or mock table were put back into home position. Study personnel entered the scanner room and collected the sample, avoiding any movements for the subjects. All saliva samples were stored at $-20{ }^{\circ} \mathrm{C}$ until being assayed at the end of the study. Analysis of cortisol concentrations was carried out using a commercial chemiluminescence immuno assay (CLIA; IBL-Hamburg, Hamburg, Germany) with a lower detection limit of $0.41 \mathrm{nmol} / \mathrm{l}$. Intraand inter-assay coefficients were below 9\%. All samples were run in duplicate in the same assay (Dressendorfer et al., 1992). Samples of one subject were run on the same kit. Salivary cortisol of one subject could not be determined, because too little saliva was available and a single salivary cortisol sample was missing for another subject. In addition to profile plots of cortisol changes of the entire lab visit, we calculated baseline to peak values (BL-Peak) for cortisol by subtracting the mean of T1 and T2 as a baseline from the maximum cortisol level during the scanner training session (T3-T6). Further, subjects exceeding cortisol BL-Peak values of $2.5 \mathrm{nmol} / 1$ were classified as responders (Wust et al., 2000).

\subsection{Statistical analysis}

Demographics, state and trait baseline characteristics between groups were tested using $\chi^{2}$ or one-way ANOVAs. Since the MRI group exhibited higher claustrophobia than the other groups the CLQ value was introduced as a covariate in all subsequent analyses. Mood and 
endocrine changes in the experimental conditions were tested using repeated measures analyses of covariance (ANCOVA) (within-subject factors ,day“ and ,sampling point“ and between-subject factor ,experimental condition“) and subsequent pairwise comparisons. As indicated by the Kolmogorov-Smirnov test (all p's > 0.09), cortisol values did not differ significantly from the normality distribution. The course of cortisol changes in responders and non-responders was tested using repeated measures analyses of covariance (ANCOVA) (within-subject factors ,sampling point“ and between-subject factor „cortisol response“) and subsequent pairwise comparisons. Greenhouse-Geisser adjustments were applied when appropriate. Group differences in BL-Peak values were tested using a two factorial ANCOVA employing the within-subjects factor „day“ and the between-subjects factor ,group“. The number of cortisol responders vs. non-responders was analyzed using a global Wald test based on general equation estimations (GEE). Differences between responders (R) and nonresponders (NR) in psychological trait and state variables were tested by t-tests. Further, we tested whether being confronted with the scanner on the first day (MRI group) vs. on the second day (lab and mock group) may alter stress reactions. Reactions towards the firsttime scanner testing were compared between groups using ANCOVAS on initial subjective ratings during the habituation task and cortisol BL-Peak values. Finally, an exploratory analysis was conducted on the relationship between subjective ratings and cortisol responding (separately for each day) using Pearson's correlations. An a level of $\mathrm{p} \leq 0.05$ indicated statistical significance. All analyses were carried our using PASW 17.0.

\section{Results}

\subsection{Sample characteristics}

Sample characteristics are given in Table 1. While the three groups were comparable regarding gender, age, and general anxiety as well as affectivity, a trend for higher CLQ values (MRI > mock, lab, $\mathrm{p}<0.050)$ and higher nervousness at day $1(\mathrm{MRI}>$ mock, $\mathrm{p}<$ 0.050 ) was observed in the MRI group. CLQ values were included as a covariate in all subsequent analyses in order to control for a priori differences between groups in claustrophobic anxiety.

\subsection{Subjective distress}

Between-session changes. Groups were comparable in initial subjective reactions towards their first-time scanner experience, meaning that participating in a factual fMRI experiment on the first (MRI) or second (mock, lab) day had no influence on subjective ratings. Significant between-session changes were however observed as a function of training environment. Mock and MRI groups showed significantly increased subjective distress on day 1 when compared to the lab group (interaction effect day $\times$ experimental condition for valence: $F(2,58)=7.574, p=0.001$; for arousal: $F(2,58)=19.110, p<0.001$; for anxiety: $\mathrm{F}(2,58)=6.848, \mathrm{p}=0.002$; pairwise comparisons day 1 : valence: mock $<$ lab, $\mathrm{p}<0.050$; arousal: MRI, mock - > lab, $\mathrm{p}<0.010$; anxiety: MRI $>$ lab, $\mathrm{p}<0.010)$. Distress decreased in the mock and MRI groups from day 1 to day 2, while increasing in the lab group (mock group: valence day $1<$ day $2, \mathrm{p}<0.010$; arousal day $1>$ day $2, \mathrm{p}<0.010$; MRI group: arousal day $1>$ day $2, \mathrm{p}<0.001$; anxiety day $1>$ day $2, \mathrm{p}<0.010$; lab group: valence day $1>$ day 2 , $\mathrm{p}<0.050$; arousal day $1<$ day $2, \mathrm{p}<0.010$; anxiety day $1<$ day $2, \mathrm{p}<0.050$; see Fig. 2 for details). 
Within-session changes. As indicated by the main effect of course, significant changes over time were observed for valence, arousal, and anxiety ratings (main effect course for valence: $\mathrm{F}(3,153)=3.757, \mathrm{p}=0.016$; arousal: $\mathrm{F}(2,138)=8.385, \mathrm{p}<0.001$; anxiety: $\mathrm{F}(3,174)=2.656$, $\mathrm{p}=0.050$ ). At the end of the habituation task negative mood (lower valence values) increased, but arousal and anxiety significantly decreased during the first half (valence: T1, 2, 3, $>\mathrm{T} 4, \mathrm{p}$ $>0.050$; arousal: $\mathrm{T} 1>2>3,4, \mathrm{p}<0.010$; anxiety: $\mathrm{T} 1>2,3,4, \mathrm{p}<0.001)$. We furthermore observed a significant interaction effect between course and day for valence, but not for arousal and anxiety ratings, pointing towards differential habituation profiles for valence ratings: while on day 1 valence decreased, values remained stable during day 2 (interaction effect course $\times$ day: valence: $\mathrm{F}(3,174)=3.922, \mathrm{p}=0.010$; arousal: $\mathrm{F}(3,148)=0.879, \mathrm{p}=$ 0.439 ; anxiety: $\mathrm{F}(2,128)=0.488, \mathrm{p}=0.634$; valence day $1: \mathrm{T} 1,2>\mathrm{T} 4, \mathrm{p}<0.050$; see Fig. 3 for details). No other main or interaction effects were observed.

\subsection{Cortisol responses}

Between-session changes. When testing for the influence of gender, no main or interaction effects were observed on cortisol responding, so that this variable was not further incorporated in the following analyses. Cortisol reactivity as indexed by BL-Peak values was comparable between groups for their respective first-time fMRI participation (MRI: day 1; mock, lab: day 2). The cortisol profile covering the entire experiment is shown in Fig. 4. We observed a significant interaction between day and experimental condition. This was driven by higher cortisol levels in general, but not specifically during scanner exposure, in the MRI group during day 1 compared to the mock and lab condition. Values generally declined from day 1 to day 2 (interaction effect day $\times$ experimental condition: $F(2,56)=4.542, p=0.015$; day 1: MRI $>$ mock, lab, $p<0.050$; MRI: day $1>$ day $2, p<0.050)$. In contrast, BL-Peak values during scanner exposure indicated a main effect of day with rising values during day 2 in the entire sample (main effect of day: $\mathrm{F}(1,57)=5.156, \mathrm{p}=0.027$; day $1<$ day $2, \mathrm{p}<0.01$, see Fig. 5). No main or interaction effects for experimental condition and day were detected. Applying a global Wald test for changing frequencies in responder rates (BL-Peak values exceeding $2.5 \mathrm{nmol}$ ), we also detected a main effect of day evidencing higher responder rates on day 2 (main effect day: $\chi 2(1)=5.938, p=0.015$ ). Further, this effect was driven by increased responder rates in the mock group and a non-significant trend for the MRI group, but not the lab group, on day 2 (interaction effect day $\times$ experimental condition: $\chi 2(5)=$ $14.459, \mathrm{p}=0.013$; mock: day $1<$ day $2, \mathrm{p}=0.046$; MRI: day $1<$ day $2, \mathrm{p}=0.064$ see Table 2).

Within-session changes. As indicated by a main effect of course for the cortisol profile, we observed falling values over time on both days (main effect course: $\mathrm{F}(2,134)=15.759, \mathrm{p}<$ 0.001 ; T1 $>5,6, \mathrm{p}<0.001$; T2 $>3,4,5,6, \mathrm{p}<0.050$; T3, $4>5,6, \mathrm{p}<0.001 ; \mathrm{T} 5>6, \mathrm{p}<$ $0.001)$. No evidence was found for rises in cortisol within the time window of the scanner session, between experimental conditions, days or as an interaction of these factors for the analysis of profile plots. On average, BL-Peak values were in the negative range for all groups on day 1 (for between-session effects on day 2 see above), supporting the notion of missing reactions towards the scanner in the majority of subjects. Response rates on day 1 however indicated that $19 \%$ of subjects showed a rise in cortisol exceeding $2.5 \mathrm{nmol}$ with comparable rates for the three groups (see Table 2; for between-session changes see above).

Characterization of cortisol responders and non-responders. As indicated by a significant interaction effect of course and cortisol response for both testing days, R and NR groups differed significantly in cortisol values during the training session, but not during baseline measures T1 and T2 (day 1: interaction effect course $\times$ cortisol response: $F(3,161)=16.288, p$ 
$<0.001$; T3, 4, 5, 6: R $>$ NR, $\mathrm{p}<0.001$; day 2: interaction effect course $\times$ cortisol response: $\mathrm{F}(2,147)=24.470, \mathrm{p}<0.001 ; \mathrm{T} 3: \mathrm{R}>\mathrm{NR}, \mathrm{p}<0.05, \mathrm{~T} 4,5,6: \mathrm{R}>\mathrm{NR}, \mathrm{p}<0.001)$. All subjects in the MRI and Mock condition that were classified as responders during day 1 were also responders during day 2 . In the lab condition, three responders from day 1 did not respond during day 2 . Although responders reported being initially more nervous on day 1 (MDMQ ratings), Responder and non-responder groups were comparable regarding gender, age, and psychological trait variables (see Supplemental Table S1).

Relationship between subjective distress and cortisol responding. On day 1, higher cortisol BL-Peak values were associated with higher initial reports on arousal (min 01: $r=0.260, p=$ $0.041)$ and lower reports on valence ratings during min 04 and 08 (min 04: $\mathrm{r}=-0.274, \mathrm{p}=$ 0.031 ; $\min 08: \mathrm{r}=0.305, \mathrm{p}=0.016)$. No significant correlations were observed during day 2.

\section{Discussion}

The aim of the present study was to explore the stress eliciting properties of an MRI environment on healthy, scanner- naïve subjects and the efficiency of three training conditions (MRI, mock, lab) in reducing stress reactions towards the scanner. The following main findings were observed: on a subjective level MRI and mock scanner training, but not the lab training were successful in reducing subjective distress towards the scanner. In contrast, no such between-session habituation effects were observed for cortisol responding: BL-Peak values generally increased during day 2 , and the percentage of cortisol responders particularly rose in the mock and (as a trend) MRI group. Further, we observed a within-session habituation of subjective arousal and anxiety during both days and irrespective of training condition. Responders and non-responders did not differ in sociodemographic or psychological trait variables. Supporting our first hypothesis, subjective distress as evidenced by lower valence, higher arousal and anxiety ratings were observed during day 1 in the MRI and mock groups compared to the lab group. From this it can be followed that both the MRI and the mock environment were successful in increasing subjective levels of distress as compared to the lab control condition. Findings are in line with observations that the mock can elicit claustrophobic reactions in college students (McGlynn et al., 2003) and can thus act as a scanner surrogate.

Conforming to our second hypothesis, MRI and mock training were followed by reduced levels of subjective distress on day 2, while rising in the lab control condition. These results correspond to previous findings from Chapman et al. (2010) reporting higher anxiety levels during the first compared to a subsequent scan. Hence, findings indicate the suitability of both training conditions for habituating subjects' subjective distress to an MRI assessment. Employing a comparative design we can conclude that effects are specific for a training in a scanner environment, since the lab control group showed rising distress levels when confronted with the scanner (day 2). Findings are thus not likely to be explained by a general habituation due to the repeated measurement design per se.

In line with our third hypothesis we observed decreasing arousal and anxiety levels throughout the habituation task on day 1 in all three groups. In contrast, valence ratings decreased (more bad mood) by the end of the habituation on day 1, but stabilized on day 2 . Obviously, subjective distress was best reflected by arousal and anxiety ratings, while valence ratings may instead have indicated different processes such as boredom that could have increased at the end of the habituation task. The fact that habituation of subjective distress 
was observed irrespective of the training condition could indicate that initially elevated distress may occur as a function of the novel experimental setting in general. Thus, it could be assumed that habituating subjects to the experimental environment may hold an advantage not only for MRI research settings. Contrary to our expectations, however, habituation effects were observed also on day 2 , indicating that the MRI and mock training had no beneficial effect on within-session habituation on the second day. Although the general level of distress declined in both groups (see above), acclimatization towards the scanner environment was nevertheless present during the second testing day. One could hence conclude that irrespective of previous mock or scanner experience subjective distress is elevated at the beginning of an (fMRI) experiment and that implementation of a short habituation allows for the reduction of state anxiety effects. Stable arousal and anxiety levels could be achieved, thus minimizing interaction with specific kinds of tasks such as affective processing, mood induction procedures or resting state measurements (see Chapman et al., 2010). It should be noted, however, that within-session habituation effects could decrease with the number of repetitions (e.g. with increasing scanner experience). For practical reasons we implemented a two day testing design. It remains to be evaluated how many training units would be necessary to completely abolish within-session habituation of anxiety and arousal. Since the amount of previous scanner experience may vary across subjects we nevertheless recommend inclusion of a short habituation phase prior to the actual scan. Regarding the time course of habituation, stable arousal and anxiety values were already obtained after approximately eight min in the present study.

Contrary to findings on subjective distress, HPA axis reactivity contradicted our hypotheses. Profile plots on cortisol evidenced higher levels in the MRI group on day 1, but these were not confined to the time window of the scanner training session. BL-Peak values focussing on reactivity during training exposure indicated a pronounced increase in all subjects on day 2 , and responder analyses further pointed towards a rise in response rates particularly in the MRI and mock group. These findings could indicate a sensitization rather than a habituation of cortisol reactivity that is particularly driven by those groups that had previous experience in a scanner environment (e.g. MRI or mock). Findings are moreover in line with those from Eatough et al. (2009) reporting that steeper declines in cortisol during mock scanning actually predicted higher responses to the MRI scan. We observed an apparent dissociation between subjective and endocrine stress parameters: although subjective distress could be reduced by a training session in a scanner environment, endocrine stress reactions showed an inverse reaction, presumably a sensitization, after repeated exposure to the scanner environment. Cortisol response rates under first time testing have been reported for approx. $12 \%$ of scanner naïve subjects in a previous independent study conducted in our lab (Muehlhan et al., 2011). After second-time testing, rates averaged at $32 \%$ in the present study which is a rather pronounced rise compared to the first day (19\%), as well as to our previous findings. Scanner training does not appear to reduce endocrine responding; instead, it could result in unintended sensitization effects. The observed dissociation between subjective and endocrine stress parameters furthermore leads to the conclusion that recording mood ratings or subjective levels of distress may result in an incomplete or even biased picture of scanner-related stress effects. Depending on the response system of interest and its putative interaction with neurohormonal activation, recording of endocrine parameters could improve controlling these unintended effects.

Present findings should be discussed within the limitations of the study. The MRI group exhibited higher claustrophobic fear than the other groups. Since subjects were randomly assigned to their respective condition, we assume that this effect was random rather than reflecting a condition-related bias. On the other hand, the CLQ was completed by the end of 
the second day. In light of our cortisol findings that indicate the presence of sensitization effects, repeated exposure to the MRI in this group might have also contributed to elevated anxiety levels post hoc. As has been previously shown (Lueken et al., 2011) claustrophobic anxiety as a trait factor accounted for differences in MRI-related state anxiety, thus requiring to control for this potential confounder statistically. Reanalyses without consideration of this covariate yielded comparable results on within- and between-session habituation. Unlike Chapman et al. (2010) we did not track changes in subjective distress across the entire scanning session, but only during the habituation task (comparable to a first fMRI task). Anxiety levels have been reported to further vary during a prolonged scanner session, and it may be a valuable additional approach to record (physiological and subjective) stress levels during the scanner session in order to introduce them as covariates into the fMRI analysis (Chapman et al., 2010; Muehlhan et al., 2011). We also acknowledge that findings are based on a sample of young and healthy volunteers. Particularly vulnerable patient groups such as anxiety patients are likely to exhibit more pronounced subjective distress than healthy controls (Lueken et al., 2011). Further, some disorders that are increasingly studied using fMRI such as major depression, panic disorder or posttraumatic stress disorder are associated with altered HPA axis function (Holsboer, 2001; Yehuda, 2006; Petrowski et al., 2010). Thus, relationships between scanner-related distress and functional activation patterns of interest are not likely to follow the same relationship in patients as in healthy controls. It could moreover be hypothesized whether state anxiety and trait features of the pathological condition could interact in a highly specific way, and studies on patient groups are needed to elucidate this question. Finally, the suitability of the mock scanner as a surrogate might depend on its actual arrangement, the instructions given, and the conditions used to simulate an MRI scan. Thus, results might not be generalized to all forms of mock scanners. It still remains to be examined which features are essential during the mock scan to induce a stress level comparable to the actual MRI scan.

The use of fMRI might be considered as the most significant methodological innovation in psychological research during the last 20 years and research will very likely continue to grow in the coming years. These developments prompt the need to study central methodological questions in order to adequately interpret the resulting data, for example the interaction between stress-eliciting properties of the scanner and functional activation patterns of interest. Here, we were able to demonstrate in a comparative study that mock and MRI training on the one hand successfully reduce subjective distress in a subsequent scanner session. On the other hand, repeated experience in an MRI environment could also induce a sensitization effect on endocrine stress levels. Subjective distress can further be stabilized by acclimating subjects to the environment prior to the fMRI assessment, employing a short habituation phase. If replicated, present findings should be particularly considered by researchers employing repeated measurement designs where subjects are exposed to a scanner more than once. Confirming previous results (Muehlhan et al., 2011), cortisol responders could also not be identified via psychological trait characteristics in the present sample, making it difficult to exclude cortisol responders a priori. Regarding the complex interaction between scannerrelated subjective distress, endocrine stress parameters and neural activations of interest, a multimodal assessment of potential confounders that can be included as covariates into the fMRI model is recommended. 

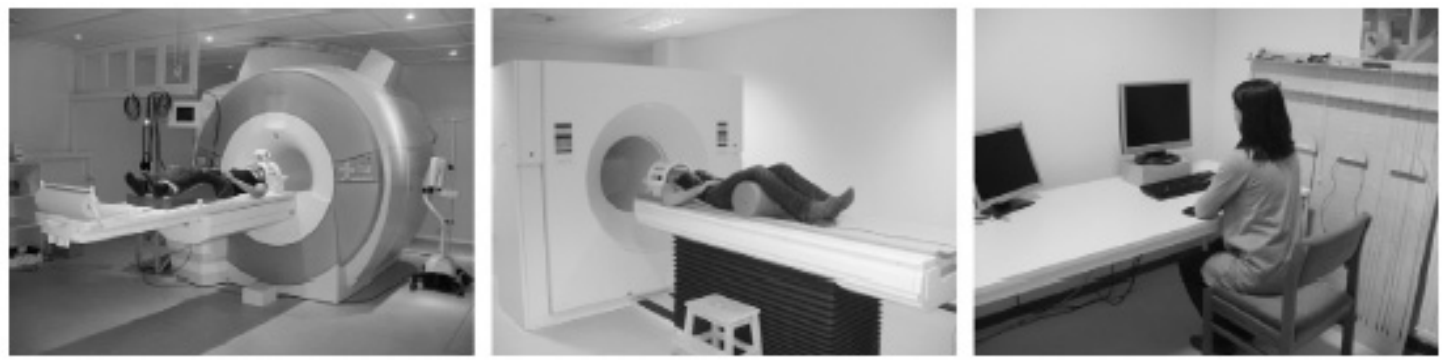

Figure 1 Visualization of the MRI, mock, and lab training environment.
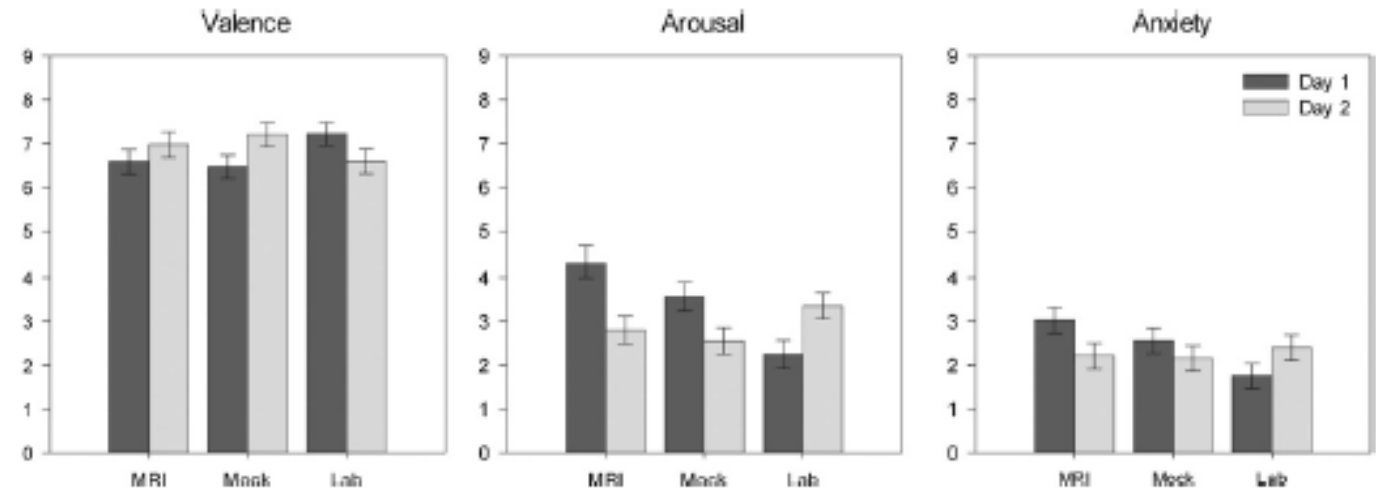

Figure 2 Between-session changes in subjective distress from day 1 to day 2, separately displayed for the training conditions (error bars indicate the standard error of mean). For valence, higher values indicate positive mood, while for arousal and anxiety, higher values indicate higher distress. MRI: MRl training condition; mock: mock training condition; lab: lab training condition (statistics on significant differences are given in the text).

Valence

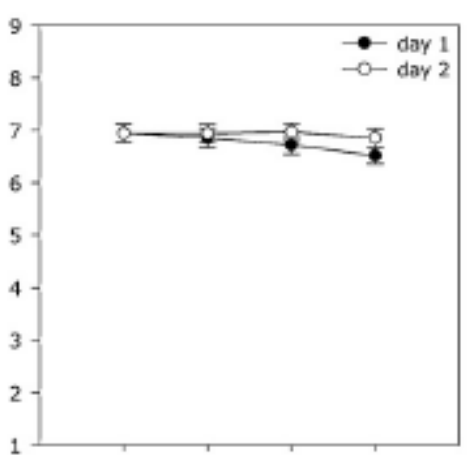

$\min 00 \min 04 \min 08 \min 12$ habituation task
Arousal

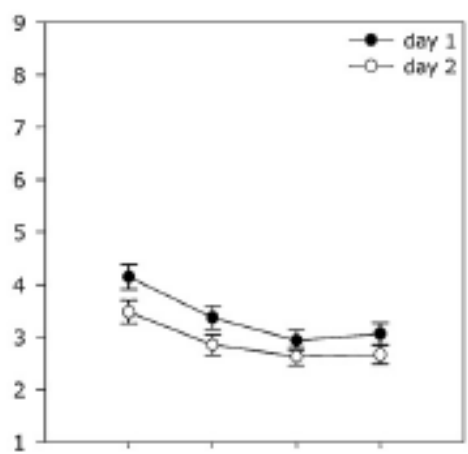

min $00 \min 04 \min 08$ min 12

habituation task
Anxiety

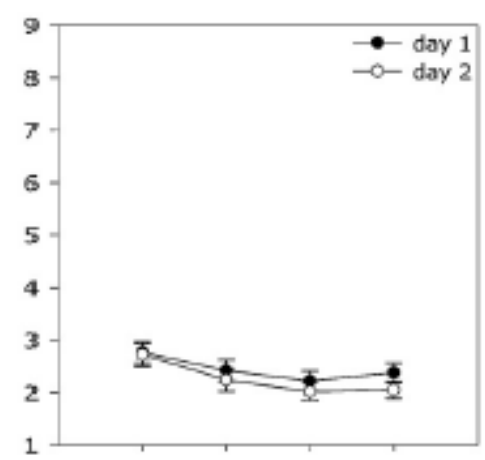

$\min 00 \min 04 \min 08 \min 12$

habituation task

Figure 3 Within-session changes in subjective distress during the habituation task, separately displayed for day 1 and day 2 (error bars indicate the standard error of means). For valence, higher values indicate positive mood, while for arousal and anxiety, higher values indicate higher distress (statistics on significant differences are given in the text). 

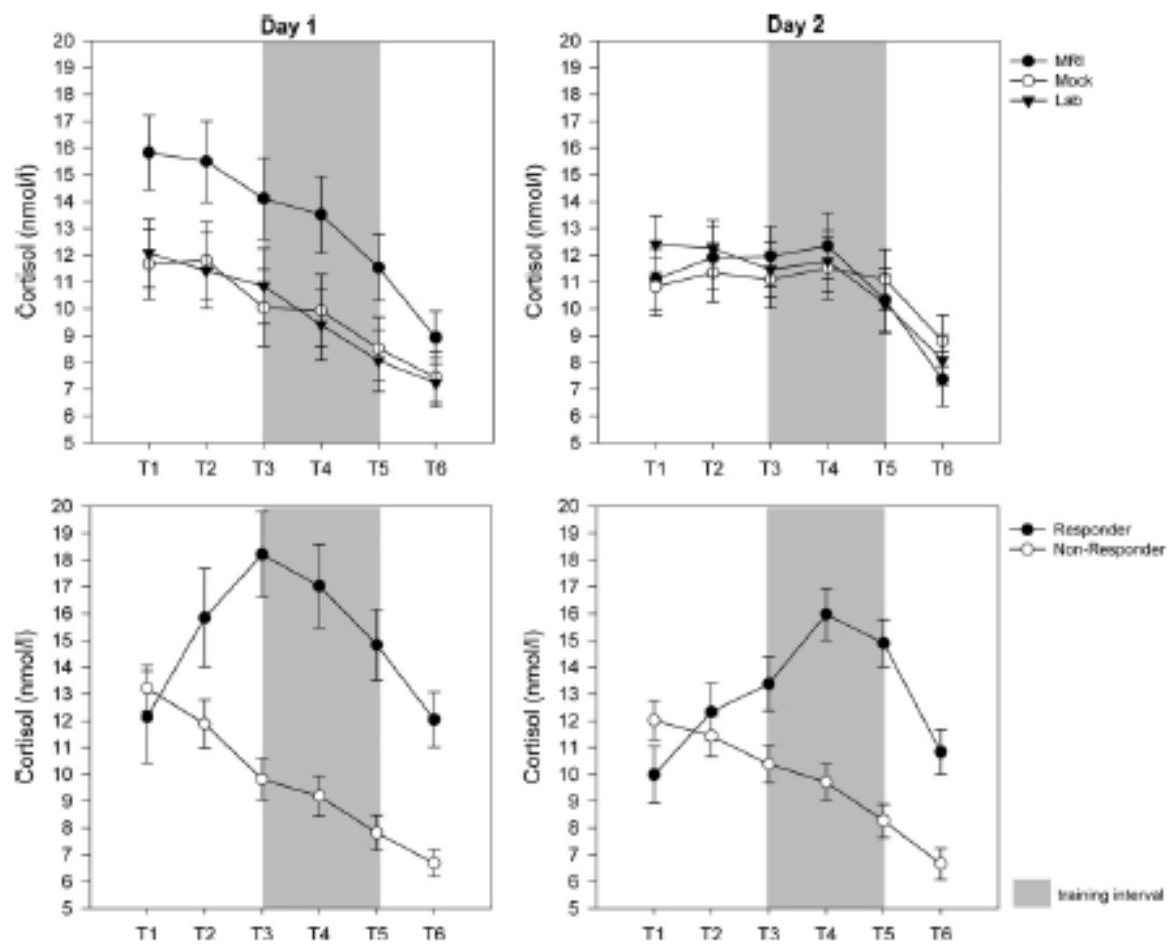

Figure 4 Cortisol profile plots covering the entire experimental session for day 1 and day 2, separately displayed for training conditions (upper half) and for cortisol response groups (lower half; error bars indicate the standard error of means). MRI: MRI training condition; mock: mock training condition; lab: lab training condition. Assessments were conducted after the informed consent (min 10; T1), after working memory-training session ( $\min 35 ; \mathrm{T} 2$ ), immediately before the habituation task ( $\min 55 ; \mathrm{T} 3$ ), after the habituation task ( $\min 70 ; \mathrm{T4}$ ), after the working memory task ( $\min 87 ; \mathrm{T}$ ), after completion of the questionnaires (min 110; T6). Statistics on significant differences are given in the text.

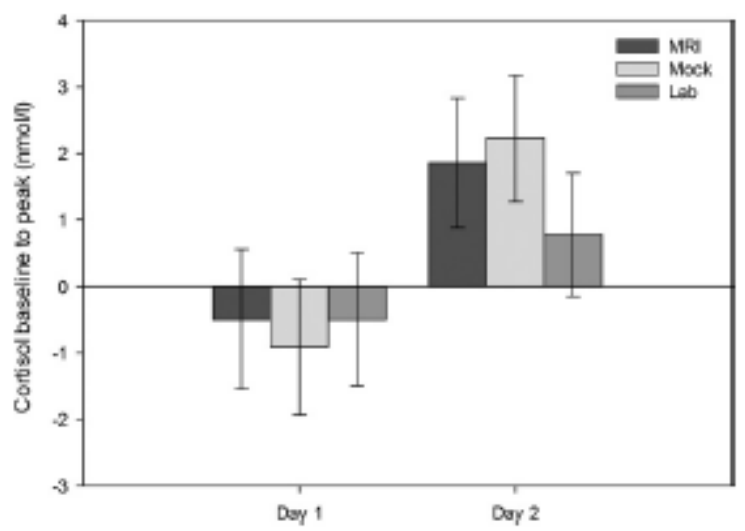

Figure 5 Baseline to peak values reflecting cortisol reactivity during the training of day 1 and day 2 , separately displayed for the training conditions (error bars indicate standard error of means). We averaged $T 1$ and $T 2$ as baseline and used the maximum during the scanner training session (T3-T6) as peak (statistics on significant differences are given in the text). 
Table 1 Sample characteristics. Means (SD) except where noted.

\begin{tabular}{|c|c|c|c|c|c|c|}
\hline & MRI $(n=20)$ & Mock $(n=22)$ & Lab $(n=21)$ & Total $(n=63)$ & $\chi^{2} / F(d f)$ & $p$ \\
\hline \multicolumn{7}{|c|}{ Demographic characteristics } \\
\hline Male $(\%)$ & $9(45)$ & $12(55)$ & $11(52)$ & $34(54)$ & $1.50(2)$ & 0.473 \\
\hline Age & $22.84(2.94)$ & $23.59(2.97)$ & $24.62(3.76)$ & $23.70(3.28)$ & $1.54(2,62)$ & 0.223 \\
\hline \multicolumn{7}{|c|}{ Trait and state affectivity } \\
\hline ASI & $14.25(4.98)$ & $15.55(7.13)$ & $12.57(5.08)$ & $14.14(5.88)$ & $1.39(2,60)$ & 0.256 \\
\hline $\mathrm{BD} I \mathrm{I}$ & $4.05(2.86)$ & $4.71(5.41)$ & $6.76(5.42)$ & $5.19(4.81)$ & $1.84(2,59)$ & 0.168 \\
\hline CLQ & $21.75(15.40)$ & $14.19(6.97)$ & $14.33(10.37)$ & $16.68(11.73)$ & $2.94(2,59)$ & 0.061 \\
\hline STAI & $37.00(6.68)$ & $37.45(8.56)$ & $35.71(6.11)$ & $36.73(7.15)$ & $0.33(2,60)$ & 0.719 \\
\hline MDMQ1_GB & $17.15(2.11)$ & $18.00(1.38)$ & $17.62(1.43)$ & $17.60(1.67)$ & $1.37(2,62)$ & 0.261 \\
\hline MDMQ1_AT & $17.35(1.63)$ & $17.00(2.07)$ & $17.10(2.53)$ & $17.14(2.09)$ & $0.15(2,62)$ & 0.860 \\
\hline MDMQ1_CN & $14.90(3.21)$ & $16.59(1.62)$ & $16.38(2.44)$ & $15.98(2.56)$ & $2.83(2,62)$ & 0.067 \\
\hline MDMQ2_GB & $17.20(2.28)$ & $17.59(2.04)$ & $17.95(1.53)$ & $17.59(1.96)$ & $0.75(2,62)$ & 0.479 \\
\hline MDMQ2 AT & $17.15(2.28)$ & $16.86(2.40)$ & $17.10(2.68)$ & $17.03(2.42)$ & $0.08(2,62)$ & 0.922 \\
\hline MDMQ2 CN & $16.70(2.45)$ & $16.95(2.30)$ & $17.38(2.13)$ & $17.02(2.28)$ & $0.46(2,62)$ & 0.632 \\
\hline
\end{tabular}

ASI: Anxiety Sensitivity Index; BDI II: Beck Depression Inventory; CLQ: Claustrophobia Questionnaire; STAI: State-Trait Arxiety Inventory: trait version; MDMQ1: Multidimensional Mood State Questionnaire on day 1; MDMQ2: Multidimensional Mood State Questionnaire on day 2; GB: Good Mood-Bad Mood; AT: Awake-Tired; CN: Calm-Nervous.

Table 2 Number of cortisol responders and non-responders on both testing days.

\begin{tabular}{|c|c|c|c|c|}
\hline & \multicolumn{2}{|l|}{ Day 1} & \multicolumn{2}{|l|}{ Day 2} \\
\hline & Responder & Non-responder & Responder & Non-responder \\
\hline MRI $(n=20)$ & $5(25 \%)$ & $15(75 \%)$ & $8(40 \%)$ & $12(60 \%)$ \\
\hline Mock $(n=21)$ & $2(10 \%)$ & $19(90 \%)$ & $6(29 \%)$ & 15 (71\%) \\
\hline Lab $(n=21)$ & $5(24 \%)$ & $16(76 \%)$ & $6(29 \%)$ & $15(71 \%)$ \\
\hline Total $(n=62)$ & $12(19 \%)$ & 50 (81\%) & $20(32 \%)$ & $42(68 \%)$ \\
\hline
\end{tabular}

MRI: MRI training condition; mock: mock training condition; lab: lab training condition; responder: baseline to peak value >2.5 nmol/L.

\section{Role of funding source}

The study was partly funded by internal grant funding of the Department of Psychology, Technische Universität Dresden (Germany). The Technische Universität Dresden had no further role in study design; in the collection, analysis and interpretation of data; in the writing of the report; and in the decision to submit the paper for publication.

\section{Conflict of interest}

U. Lueken, M. Muehlhan, R. Evens and C. Kirschbaum report no conflict of interest. H.-U. Wittchen has served as a general consultant (non-product related) for Pfizer, Organon, Servier, Essex Pharma and has received grant funding for his institution from Sanofi Aventis, Pfizer, Lundbeck, Novartis, Essex Pharma, Servier and Whyet.

\section{Acknowledgements}

No acknowledgements.

\section{Appendix A. Supplementary data}

Supplementary data associated with this article can be found, in the online version, at doi:10.1016/j.psyneuen. 2012.01.003. 


\section{References}

- Chapman, H.A., Bernier, D., Rusak, B., 2010. MRI-related anxiety levels change within and between repeated scanning sessions. Psychiatry Res. Neuroimaging 182, 160-164.

- Corbett, B.A., Mendoza, S., Abdullah, M., Wegelin, J.A., Levine, S., 2006. Cortisol circadian rhythms and response to stress in children with autism.

Psychoneuroendocrinology 31, 59-68.

- De Bie, H.M.A., Boersma, M., Wattjes, M.P., Adriaanse, S., Vermeulen, R.J., Oostrom, K.J., Huisman, J., Veltman, D.J., Delemarre-Van de Waal, H., 2010. Preparing children with a mock scanner training protocol results in high quality structural and functional MRI scans. Eur. J. Pediatr. 169, 1079-1085.

- Dewey, M., Schink, T., Dewey, C.F., 2007. Claustrophobia during magnetic resonance imaging: cohort study in over 55,000 patients. J. Magn. Reson. Imaging 26, 13221327.

- Dressendorfer, R.A., Kirschbaum, C., Rohde, W., Stahl, F., Strasburger, C.J., 1992. Synthesis of a cortisol-biotin conjugate and evaluation as a tracer in an immunoassay for salivary cortisol measurement. J. Steroid. Biochem. Mol. Biol. 43, 683-692.

- $\quad$ Eatough, E.M., Shirtcliff, E.A., Hanson, J.L., Pollak, S.D., 2009. Hormonal reactivity to MRI scanning in adolescents. Psychoneuroendocrinology 34, 1242-1246.

- Fishbain, D.A., Goldberg, M., Labbe, E., Zacher, D., Steelerosomoff, R., Rosomoff, H., 1988. Long-term claustrophobia following magnetic resonance imaging. Am. J. Psychiatry 145, 1038-1039.

- Grey, S.J., Price, G., Mathews, A., 2000. Reduction of anxiety during MR imaging: a controlled trial. Magn. Reson. Imaging 18, 351-355.

- Hautzinger, M., Keller, F., Kühner, C., 2006. Beck Depressionsinventar-II. Harcourt, Frankfurt/Main.

- Holsboer, F., 2001. Stress, hypercortisolism and corticosteroid receptors in depression: implications for therapy. J. Affect. Disord. 62, 77-91.

- Hoyer, J., Markgraf, J., 2003. Angstdiagnostik. Grundlagen und Testverfahren. Springer, Berlin.

- Laux, L., Glanzmann, P., Schaffner, P., Spielberger, C.D., 1981. State-TraitAngstinventar (STAI). Theoretische Grundlagen und Handanweisung. Beltz, Weinheim.

- Lueken, U., Muehlhan, M., Wittchen, H.U., Kellermann, T., Reinhardt, I., Konrad, C., Lang, T., Wittmann, A., Strohle, A., Gerlach, A.L., Ewert, A., Kircher, T., 2011. (Don't) panic in the scanner! How panic patients with agoraphobia experience a functional magnetic resonance imaging session Eur. Neuropsychopharmacol. 21, 516525.

- McGlynn, F.D., Karg, R., Lawyer, S.R., 2003. Fear responses to mock magnetic resonance imaging among college students: toward a prototype experiment. J. Anxiety Disord. 17, 335-347.

- McGlynn, F.D., Smitherman, T.A., Hammel, J.C., Lazarte, A.A., 2007. Component fears of claustrophobia associated with mock magnetic resonance imaging. J. Anxiety Disord. 21, 367-380.

- Muehlhan, M., Lueken, U., Wittchen, H.U., Kirschbaum, C., 2011. The scanner as a stressor: evidence from subjective and neuroendocrine stress parameters in the time course of a functional magnetic resonance imaging session. Int. J. Psychophysiol. 79, 118-126. 
- Peters, S., Cleare, A.J., Papadopoulos, A., Fu, C.H., 2011. Cortisol responses to serial MRI scans in healthy adults and in depression. Psychoneuroendocrinology 36, 737741.

- Petrowski, K., Herold, U., Joraschky, P., Wittchen, H.U., Kirschbaum, C., 2010. A striking pattern of cortisol non-responsiveness to psychosocial stress in patients with panic disorder with concurrent normal cortisol awakening responses. Psychoneuroendocrinology 35, 414-421.

- $\quad$ Quirk, M.E., Letendre, A.J., Ciottone, R.A., Lingley, J.F., 1989. Evaluation of 3 psychological interventions to reduce anxiety during MR imaging. Radiology 173, 759-762.

- Radomsky, A.S., Rachman, S., Thordarson, D.S., McIsaac, H.K., Teachman, B.A., 2001. The Claustrophobia Questionnaire. J. Anxiety Disord. 15, 287-297.

- Spiegelhalder, K., Hornyak, M., Kyle, S.D., Paul, D., Blechert, J., Seifritz, E., Hennig, J., van Elst, L.T., Riemann, D., Feige, B., 2009. Cerebral correlates of heart rate variations during a spontaneous panic attack in the fMRI scanner. Neurocase 15, 527534.

- Steyer, R., Schwenkmetzger, P., Notz, P., Eid, M., 1997. Mehrdimensionaler Befindlichkeitsfragebogen. Hogrefe, Göttingen.

- Tessner, K.D., Walker, E.F., Hochman, K., Hamann, S., 2006. Cortisol responses of healthy volunteers undergoing magnetic resonance imaging. Hum. Brain. Mapp. 27, 889-895.

- Weitzman, E.D., Fukushim, D., Nogeire, C., Roffwarg, H., Gallaghe, T.F., Hellman, L., 1971. $24 \mathrm{H}$ pattern of episodic secretion of cortisol in normal subjects. J. Clin. Endocrinol. Metab. 33, 14-22.

- Wood, B.S., McGlynn, F.D., 2000. Research on posttreatment return of claustrophobic fear, arousal, and avoidance using mock diagnostic imaging. Behav. Modif. 24, 379394.

- Wust, S., Wolf, J., Hellhammer, D.H., Federenko, I., Schommer, N., Kirschbaum, C., 2000. The cortisol awakening response - normal values and confounds. Noise Health 2, 79-88.

- Yehuda, R., 2006. Advances in understanding neuroendocrine alterations in PTSD and their therapeutic implications. Ann. N. Y. Acad. Sci. 1071, 137-166. 